\title{
Efficacy of passive helmet therapy for deformational plagiocephaly: report of 1050 cases
}

\author{
Daniel E. Couture, M.D., ${ }^{1}$ John C. Crantford, M.D., ${ }^{2}$ Aravind Somasundaram, B.S., ${ }^{1}$ \\ Claire Sanger, D.O., ${ }^{2}$ Anne E. Argenta, M.D., ${ }^{3}$ and Lisa R. David, M.D. ${ }^{2}$
}

Departments of ${ }^{1}$ Neurosurgery and ${ }^{2}$ Plastic and Reconstructive Surgery, Wake Forest Baptist Medical Center, Winston-Salem, North Carolina; and ${ }^{3}$ Department of Plastic Surgery, University of Pittsburgh Medical Center, Pittsburgh, Pennsylvania

\begin{abstract}
Object. There has been a tremendous increase in the incidence of deformational plagiocephaly in children throughout the world. Therapeutic options include observation, active counterpositioning, external orthotics, and surgery. The current treatment in the US is highly debated, but it typically includes external orthotic helmets in patients with moderate to severe plagiocephaly presenting between 4 and 10 months of age or in children with significant comorbidities limiting passive (no-pressure) therapy. The present study was designed to evaluate 3 key issues: 1) the accuracy of the Argenta classification in defining a progressive degree of severity, 2) identification of an upper age limit when treatment is no longer effective, and 3) the effectiveness of an off-the-shelf prefabricated helmet in correcting deformational plagiocephaly.

Methods. An institutional review board-approved retrospective study was conducted of all patients at the authors' clinic in whom deformational plagiocephaly was assessed using the Argenta classification system over a 6-year period; the patients underwent helmet therapy, and a minimum of 3 clinic visits were recorded. Inclusion criteria consisted of an Argenta Type II-V plagiocephalic deformity. Patients' conditions were categorized both by severity of the deformity and by patients' age at presentation. Statistical analysis was conducted using survival analysis.

Results. There were 1050 patients included in the study. Patients with Type III, IV, and V plagiocephaly required progressively longer for deformity correction to be achieved than patients with Type II plagiocephaly $(53 \%, 75 \%$, and $81 \%$ longer, respectively $[\mathrm{p}<0.0001])$. This finding verified that the Argenta stratification indicated a progressive severity of deformity. No statistically significant difference in the time to correction was noted among the different age categories, which suggests that the previously held upper time limit for correction may be inaccurate. An overall correction rate to Type I plagiocephaly of $81.6 \%$ was achieved irrespective of severity and degree of the original deformity. This suggests that an inexpensive off-the-shelf molding helmet is highly effective and that expensive custom-fitted orthoses may not be necessary. The patients in the older age group ( $>12$ months) did not have a statistically significant longer interval to correction than the patients in the youngest age group $(<3$ months $)$. The mean length of follow-up was 6.3 months.

Conclusions. Patients treated with passive helmet therapy in the older age group ( $>12$ months) had an improvement in skull shape within the same treatment interval as the patients in the younger age group $(<3$ months $)$. This study supports the use of passive helmet therapy for improvement in deformational plagiocephaly in infants from birth to 18 months of age and verifies the stratification of degree of deformity used in the Argenta classification system.
\end{abstract}

(http://thejns.org/doi/abs/10.3171/2013.8.FOCUS13258)

KEY WORDS
child

$\mathrm{T}$ He American Academy of Pediatrics launched a campaign to educate the community and to promote the supine sleeping position for infants, the "Back to Sleep" campaign, ${ }^{1}$ after identifying prone sleeping as a key causative factor for sudden infant death syn-

Abbreviation used in this paper: SIDS = sudden infant death syndrome. drome (SIDS). Since the start of the campaign in 1992, there has been a documented decline in SIDS by as much as $50 \% ;{ }^{10}$ however, an unfortunate consequence has been a drastic increase in the incidence of infants diagnosed with deformational plagiocephaly, also referred to as occipital plagiocephaly. Although increased awareness may be contributing to the increased incidence, deformational plagiocephaly is a common entity that may be present in 
as many as 1 in 5 infants..$^{23}$ Deformational plagiocephaly is defined as skull asymmetry due to external forces unrelated to premature fusion of one or more of the cranial sutures. Risk factors include prematurity, breech position, assisted delivery, first-born child, maternal age (> 35 years), and male sex. ${ }^{17}$ The classic presentation of an infant with deformational plagiocephaly is unilateral flattening of the occiput. Depending on the severity of the deformity, the ipsilateral ear will be anteriorly displaced and the ipsilateral forehead prominent, thus displaying a parallelogram shape. ${ }^{20}$ In more severe cases, the malar bones will be asymmetrical, while in the most severe cases a mild constriction results in vertical skull growth and temporal bossing. Central flattening of the posterior skull with vertical growth can also occur and the frontal view of the face will appear asymmetrical as well. Patients may also present with complete flattening of the occipital area, with either lateral or cephalad prominence. Argenta et al. have described an extensive clinically reproducible classification system that greatly assists in the clinical discussion among the craniofacial surgeon, parents, and referring physicians (Fig. 1). ${ }^{2}$ Therapeutic options include observation, active counterpositioning, external orthotics, and surgery. . $^{4,8,15,18,22}$

Most craniofacial surgeons today agree that deformational plagiocephaly does not warrant surgical intervention..$^{5,15}$ Varying the infant's positioning is the key to avoiding positional cranial deformation. Avoidance of prolonged periods in car seats, swings, and other devices that minimize infant motion are important. ${ }^{6,10,17}$ Caregivers need to be educated and encouraged to provide the infant with adequate time in the prone position, or "tummy time." This provides the infant an opportunity to build the muscle strength of the arms, neck, and upper back and will help to improve the motor development that is typically slow to develop in these children. ${ }^{11}$ The remaining therapeutic options are still under investigation, as there are many differing opinions on the most effective treatment for this condition. ${ }^{6}$ The current treatment in the US includes external orthotic helmets for moderate to severe cases of deformational plagiocephaly in children between 4 and 8 months of age. ${ }^{21}$ It has long been felt that once the fontanel closes at 9-12 months of age, the window for potential treatment has passed. This idea stems from the fact that as an infant nears 1 year of age, the calvaria increases in size and thickness and the cranial base bones become deformed from the chronic effects of positional molding, making the skull less malleable to treatment with positioning or orthotics. ${ }^{16}$ One of the objectives of the present study was to evaluate the optimal time for initiating helmet therapy and to determine if deformity correction is obtainable in the older age group. The aim of the study was not to compare other treatment modalities to passive (no pressure) helmet therapy, and we acknowledge that passive helmet therapy was not shown to be more effective that other therapies.

\section{Occipital Plagiocephaly}
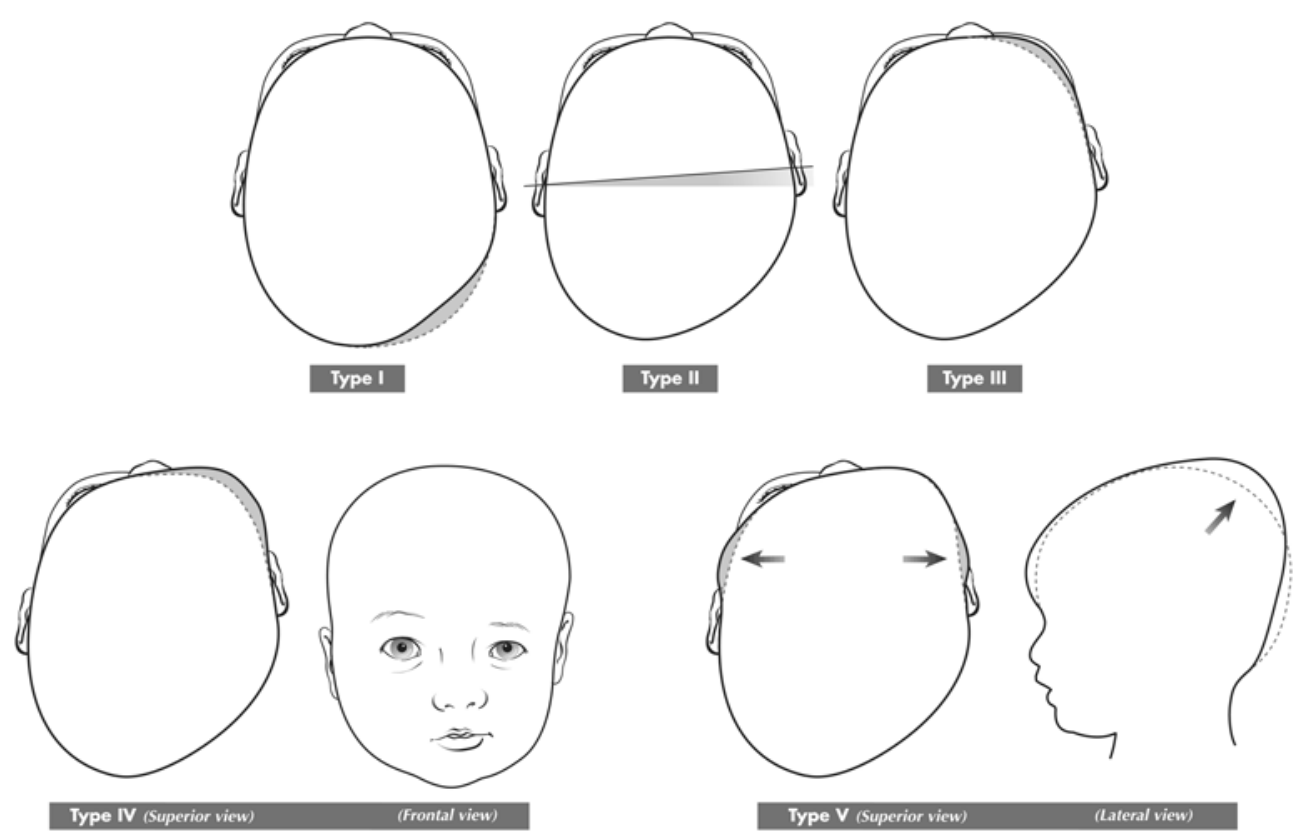

2004 WFUSM Plastic and Reconstructive Surgery Collection

FIG. 1. Sketches depicting types of deformational plagiocephaly in the Argenta classification system, in which there are 5 stages of deformity progressing from minimal to severe: Type I deformity is restricted to the back of the skull; Type II adds malposition of the affected ear; Type III adds forehead deformity; Type IV adds malar deformity; and Type V adds attempts of the brain to decompress vertically or temporally. Originally published in "A Collection of Images and Illustrations" by the Department of Plastic Surgery, Wake Forest University of Medicine. Used with permission. 


\section{Passive helmet therapy for deformational plagiocephaly}

\section{Methods}

An institutional review board-approved retrospective study was conducted at the North Carolina Center for Cleft and Craniofacial Deformities at Wake Forest University to evaluate all patients who were seen for deformational plagiocephaly and who attended at least 3 serial clinic visits during a 6-year period. The charts were reviewed and only patients who were treated with a prefabricated passive helmet (Fig. 2) were included in the study (Danmar Products). Patients treated with conservative measures are only seen twice during follow-up before having their care transferred back to their pediatrician; thus, no long-term data are available for these patients to use as controls. In all patients an Argenta classification severity level was documented from the time of diagnosis to the completion of treatment, and 3D surface laser scans were used to confirm this classification. The age at presentation, duration of treatment, and follow-up duration were recorded. Compliance with helmet use was also recorded. Inclusion criteria required the presence of an Argenta Type II-V deformity. Patients with a Type I (minimal) deformity were not included because they were treated with positioning rather than with helmets. Patients with a Type VI (central) deformity were excluded because they represent a separate group of patients. Patients' conditions were categorized both by severity of the deformity and by patients' age at presentation. The severity of plagiocephly was determined clinically by evaluating the

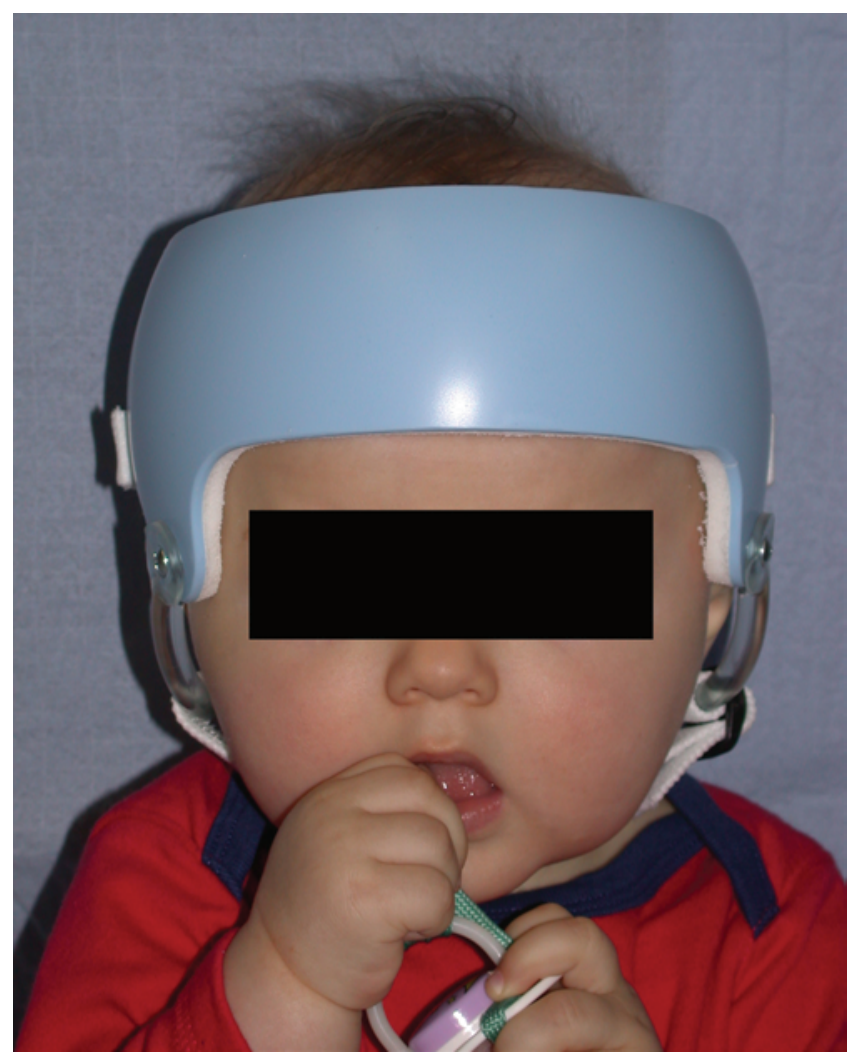

FIG. 2. Clinical illustration of the helmet on an infant. Originally published in "A Collection of Images and Illustrations" by the Department of Plastic Surgery, Wake Forest University of Medicine. Used with permission. patient from the cephalic (bird's-eye), lateral, and anterior views for shape and landmark locations. This provided a thorough evaluation to determine the severity and location of the occipital flattening, location and severity of displacement of the external auditory canals, and presence and severity of forehead prominence. The frontal view provided an assessment of the facial features to determine if there was any asymmetry.

Patients were followed up until correction was achieved (Level I or better). Three-dimensional laser scans (FastScan Cobra, Polhemus Inc.) were obtained throughout therapy and at the final visit before discontinuing therapy. Statistical analysis was conducted using survival analysis.

\section{Results}

There were 1050 patients included in the study. The mean follow-up period was 6.3 months. The greatest number of patients were classified as having an Argenta Type III deformity, and this was followed by Types IV, II, and V. The patients' ages ranged from 19 days to 21.6 months. Patients were stratified into 5 groups based on age: less than 3 months $(n=60), 4-6$ months $(n=584)$, 7-9 months $(\mathrm{n}=330), 10-12$ months $(\mathrm{n}=61)$, and greater than 12 months $(\mathrm{n}=15)$. There was an overall correction rate of $81.6 \%$ (Tables 1 and 2). Time to correction is defined as the duration in months it took to reach an Argenta classification severity level of I or 0. As seen in Fig. 3, patients with Type III, IV, and V deformational plagiocephaly required progressively longer times for correction to be achieved compared with patients with Type II deformity $(53 \%, 75 \%$, and $81 \%$ longer, respectively [p $<0.0001]$ ). There was no statistically significant difference in the time to correction among the 5 age categories, although there was a trend toward shorter time to correction in the older age groups (Fig. 4). The patients in the older age group (>12 months) did not have a statistically longer time to correction when compared with the youngest age group ( $<3$ months). The mean length of follow-up period was 6.3 months.

\section{Discussion}

In the current decade, deformational plagiocephaly has become the leading cause of abnormal skull shape in infants and has resulted in a marked increase in the awareness of this condition among the general public. The lack

TABLE 1: Summary of cases in which deformity was corrected to a Type I or Type 0 deformity by helmet therapy

\begin{tabular}{ccc}
\hline Initial Type & No. of Patients & $\begin{array}{c}\text { No. Corrected to Type 0 or } \\
\text { I Deformity (\%) }\end{array}$ \\
\hline II & 177 & $164(92.7)$ \\
III & 560 & $476(85)$ \\
IV & 265 & $194(73.2)$ \\
V & 48 & $23(47.9)$ \\
total & 1050 & $857(81.6)$ \\
\hline
\end{tabular}




\section{E. Couture et al.}

TABLE 2: Summary of cases in which deformity was corrected to Type I or Type 0 deformity stratified by age group

\begin{tabular}{lcc}
\hline Age (mos) & No. of Patients & $\begin{array}{c}\text { No. Corrected to Type 0 or } \\
\text { I Deformity (\%) }\end{array}$ \\
\hline$\leq 3$ & 60 & $56(93.3)$ \\
$4-6$ & 584 & $484(82.9)$ \\
$7-9$ & 330 & $269(81.5)$ \\
$10-12$ & 61 & $40(65.6)$ \\
$>12$ & 15 & $8(53.3)$ \\
total & 1050 & $857(81.6)$ \\
\hline
\end{tabular}

of Level I evidence has led to much debate among physicians as to whether treatment is necessary, which modalities are most effective, and at what point treatment should be optimally initiated. ${ }^{17}$ By means of a survey, Lee et al. showed that craniofacial surgeons are more likely than neurosurgeons to prescribe helmet therapy for infants presenting with deformational plagiocephaly. ${ }^{13}$ Treatment may therefore be influenced by referral patterns and the final objective of the treating physician. In general, repositioning is recommended for infants with mild to moderate asymmetry or infants younger than 4 months, while helmet therapy is recommended for infants with severe asymmetry or infants 6 months or older whose deformity has not responded to repositioning. ${ }^{6}$ Despite these general guidelines, no Level I evidence supports these recommendations. The aim of our study was to investigate if age is a factor in infants whose condition responded to helmet therapy.

There is no doubt that an abnormal or asymmetrical skull shape in an infant is of great concern to parents. In today's society abnormal facial and cranial features are associated with the supposition of functional impairment leading to social maladaptation. Greater numbers of studies are confirming the correlation between positional plagiocephaly and neurodevelopmental delay seen in fine motor skills. ${ }^{7,9,12,19}$ Whether the developmental delay or the plagiocephaly is the causative factor remains unanswered. The importance of regular repositioning and observed tummy time cannot be overstated. It is during the first few months of life that the child needs to develop the neck, back, and upper arm muscles. This is best done in the prone position and then when the infant begins to crawl. The reality of growing numbers of single-parent

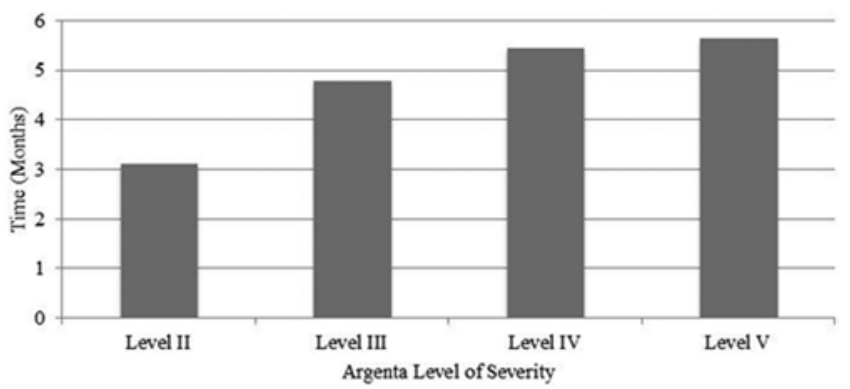

FIG. 3. Average time to correction to Argenta Level I or 0 stratified by level of severity (Argenta type) at initial presentation of patients treated with passive helmet therapy.

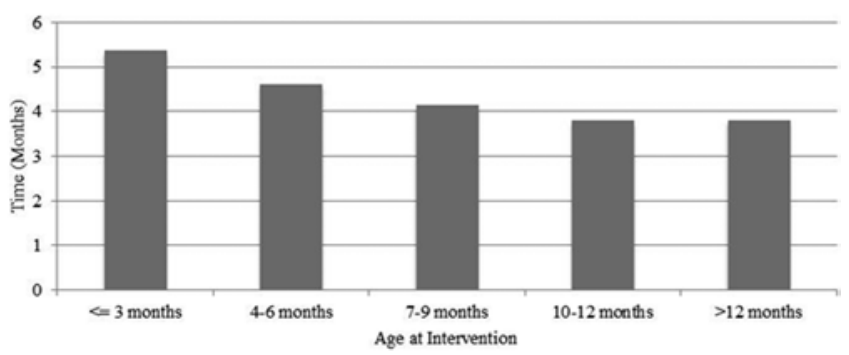

FIG. 4. Average time to correction to Argenta Level I or 0 stratified by age at initiation of treatment with passive helmet therapy.

households and dual working parents should be considered when developing treatment paradigms for infants with positional plagiocephaly. It is difficult for caregivers, often caring for multiple children, to find lengthy periods during the day to observe infants in the prone position. Considering these realities, our treatment protocol includes passive helmet therapy for children with greater than Argenta Class II deformity and active molding helmet therapy for children with Class $\mathrm{V}$ deformity who present at 9 months or older and for patients older than 18 months.

The Argenta classification system was initially proposed as a way to represent a progressive degree of deformity, with each increasing number indicating a more severe deformity. Deformities are classified on a scale from I to $\mathrm{V}$, and each progressive grade indicates more involvement of the skull or face. The classification is based on clinical observation alone and allows the practitioner to accurately quantitate the degree of deformity in any patient. ${ }^{2}$ At the initial visit, the deformity was classified based on physical observational criteria. At subsequent visits, typically 2 months apart, the classification was determined by investigators who were blinded to the previous classification. Over time, a progressive decrease in deformity level was noted in almost all the treated infants, indicating a lesser degree of observable deformity. Increases in severity index invariably coincided with failure to wear the helmet consistently. The pattern of correction observed in children who consistently wore the helmet during the treatment period indicated that the Argenta classification system correctly defines a progressive decrease of severity.

Most craniofacial surgeons have prescribed helmets based on the age of the patient. It is postulated that closure of the fontanel and progressive ossification occur as the infant ages, reducing the likelihood for correction with helmet therapy. Many surgeons feel helmet treatment after 9 months of age is unproductive, and most feel that in patients older than 12 months little hope for improvement can be expected.

The increasing incidence of older adopted children and children from rural communities with significant posterior deformities prompted treatment of older children in our clinic. It was felt that any improvement in significant asymmetries would be better than none. Our study indicated that with consistent helmet use of 23 hours per day, deformities can be corrected in children up to 18 months of age. The duration required for correction was not significantly different for children greater than 12 months 
compared with children at 3 months of age. The time to correction in children more than 12 months of age increased with the increasing Argenta class but was similar to that documented in infants treated at 3 months in the same cohort. Parental concern and rigid enforcement of compliance with helmet use may contribute to successful deformity correction in those children. Parents facing a rapidly closing treatment window may be more compliant with therapy. Within a given Argenta severity level, children undergoing helmet therapy earlier did attain a better clinical result than those who underwent helmet therapy at 12 months of age or greater. It is our opinion that earlier helmeting optimizes outcome, especially when factors such as compliance are entered into the equation. We believe our 12-month-and-older group did so well because they had very motivated parents.

The third objective of our study was to determine if a prefabricated off-the-shelf helmet was sufficient to correct positional asymmetry. Insurance companies have increasingly excluded paying for molding helmets until functional problems are documented, labeling positional plagiocephaly a cosmetic disorder.6,14 The price for orthotic devices can range from $\$ 2000$ to $\$ 4000$, precluding treatment for many children. ${ }^{3}$ In addition to high prices, the custom fabricated active molding helmets require weekly readjustments with additional time for travel and loss of work for caregivers. The helmet used in our study was prefabricated in 2 sizes and 2 colors. It is size adjustable and functions as a passive (no pressure) template that modifies the shape of the growing skull. The price is between $\$ 500$ and $\$ 600$, and visits to the clinic are bimonthly, reducing the financial and time burden caregivers face when treating their infant. None of the patients in our study developed pressure problems or helmet-related complications.

As in previous studies, successful treatment correlates with consistent wearing of the helmet. ${ }^{4}$ Our overall success rate, irrespective of the degree of deformity and age, was $82 \%$. The significant decreased orthotics cost and the need for less frequent clinic visits significantly increase the availability of treatment for these children.

\section{Conclusions}

Patients treated with passive helmet therapy in the older age group (> 12 months) have an improvement in skull shape in the same time interval as the patients in the younger age group ( $<3$ months). This study supports the use of passive helmet therapy for improvement in deformational plagiocephaly in infants from birth to 18 months of age and verifies the Argenta classification as a useful stratification of degree of deformity. No external validation methods have been done on the patients included in this study. The initial descriptive paper referenced in this study did do this to a degree. The authors acknowledge the subjective nature of the classification system but find it a reliable way for clinicians to evaluate and communicate severity levels with other care providers.

\section{Disclosure}

The authors report no conflict of interest concerning the mate- rials or methods used in this study or the findings specified in this paper.

Author contributions to the study and manuscript preparation include the following. Conception and design: David, Couture. Acquisition of data: Crantford, Somasundaram, Sanger, Argenta. Analysis and interpretation of data: David, Couture, Crantford, Somasundaram, Argenta. Drafting the article: Crantford. Critically revising the article: David, Couture. Reviewed submitted version of manuscript: David, Couture. Approved the final version of the manuscript on behalf of all authors: David. Statistical analysis: David. Administrative/technical/material support: David, Couture. Study supervision: David, Couture.

\section{References}

1. American Academy of Pediatrics Task Force on Infant Positioning and SIDS: Positioning and SIDS. Pediatrics 89:11201126, 1992 (Erratum in Pediatrics 90:264, 1992)

2. Argenta L, David L, Thompson J: Clinical classification of positional plagiocephaly. J Craniofac Surg 15:368-372, 2004

3. BlueCross BlueShield of Tennessee: Helmets or headbands for infants with irregularly shaped heads. (http://www. bcbst.com/learn/treatment-options/HelmetsHeadband.shtm) [Accessed August 13, 2013]

4. Carson BS, Muñoz D, Gross G, VanderKolk CA, James CS, Gates J, et al: An assistive device for the treatment of positional plagiocephaly. J Craniofac Surg 11:177-183, 2000

5. David DJ, Menard RM: Occipital plagiocephaly. Br J Plast Surg 53:367-377, 2000

6. Flannery ABK, Looman WS, Kemper K: Evidence-based care of the child with deformational plagiocephaly, part II: management. J Pediatr Health Care 26:320-331, 2012

7. Fowler EA, Becker DB, Pilgram TK, Noetzel M, Epstein J, Kane AA: Neurologic findings in infants with deformational plagiocephaly. J Child Neurol 23:742-747, 2008

8. Hutchison BL, Stewart AW, De Chalain TB, Mitchell EA: A randomized controlled trial of positioning treatments in infants with positional head shape deformities. Acta Paediatr 99:1556-1560, 2010

9. Hutchison BL, Stewart AW, Mitchell EA: Deformational plagiocephaly: a follow-up of head shape, parental concern and neurodevelopment at ages 3 and 4 years. Arch Dis Child 96: 85-90, 2011

10. Kattwinkel J, Hauck F, Keenan M, Malloy M, Moon R: The changing concept of sudden infant death syndrome: diagnostic coding shifts, controversies regarding the sleeping environment, and new variables to consider in reducing risk. Pediatrics 116:1245-1255, 2005

11. Kennedy E, Majnemer A, Farmer JP, Barr RG, Platt RW: Motor development of infants with positional plagiocephaly. Phys Occup Ther Pediatr 29:222-235, 2009

12. Kordestani RK, Patel S, Bard DE, Gurwitch R, Panchal J: Neurodevelopmental delays in children with deformational plagiocephaly. Plast Reconstr Surg 117:207-220, 2006

13. Lee A, Van Pelt AE, Kane AA, Pilgram TK, Govier DP, Woo AS, et al: Comparison of perceptions and treatment practices between neurosurgeons and plastic surgeons for infants with deformational plagiocephaly. Clinical article. J Neurosurg Pediatr 5:368-374, 2010

14. Littlefield TR, Kelly KM: Deformational plagiocephaly: recommendations for future research. J Prosthet Orthot 16:S59S62, 2004

15. Marchac A, Arnaud E, Di Rocco F, Michienzi J, Renier D: Severe deformational plagiocephaly: long-term results of surgical treatment. J Craniofac Surg 22:24-29, 2011

16. Pollack IF, Losken HW, Fasick P: Diagnosis and management of posterior plagiocephaly. Pediatrics 99:180-185, 1997

17. Robinson S, Proctor M: Diagnosis and management of defor- 


\section{E. Couture et al.}

mational plagiocephaly. A review. J Neurosurg Pediatr 3: 284-295, 2009

18. Rogers GF: Severe deformational plagiocephaly: long-term results of surgical treatment. J Craniofac Surg 22:30-32, 2011

19. Speltz ML, Collett BR, Stott-Miller M, Starr JR, Heike C, Wolfram-Aduan AM, et al: Case-control study of neurodevelopment in deformational plagiocephaly. Pediatrics 125: e537-e542, 2010

20. Teichgraeber JF, Ault JK, Baumgartner J, Waller A, Messersmith M, Gateño J, et al: Deformational posterior plagiocephaly: diagnosis and treatment. Cleft Palate Craniofac J 39: 582-586, 2002

21. Thompson JT, David LR, Wood B, Argenta A, Simpson J, Argenta LC: Outcome analysis of helmet therapy for positional plagiocephaly using a three-dimensional surface scanning laser. J Craniofac Surg 20:362-365, 2009

22. van Vlimmeren LA, van der Graaf Y, Boere-Boonekamp
MM, L'Hoir MP, Helders PJ, Engelbert RH: Effect of pediatric physical therapy on deformational plagiocephaly in children with positional preference: a randomized controlled trial. Arch Pediatr Adolesc Med 162:712-718, 2008

23. van Vlimmeren LA, van der Graaf Y, Boere-Boonekamp MM, L'Hoir MP, Helders PJ, Engelbert RH: Risk factors for deformational plagiocephaly at birth and at 7 weeks of age: a prospective cohort study. Pediatrics 119:e408-e418, 2007

Manuscript submitted June 14, 2013.

Accepted August 7, 2013.

Please include this information when citing this paper: DOI: 10.3171/2013.8.FOCUS13258.

Address correspondence to: Lisa R. David, M.D., Department of Plastic and Reconstructive Surgery, Wake Forest Baptist Medical Center, Winston-Salem, NC 27157. email: ldavid@wakehealth.edu. 